\title{
Development and Performance Evaluation of Fish-Type Robot using Artificial Muscles and Servo Motors as Actuators
}

\author{
Sinpei Fujiwara ${ }^{\mathrm{a},{ }^{*}}$ and Satoru Yamaguchi ${ }^{\mathrm{b}}$ \\ ${ }^{a}$ Graduate School of Engineering, Kyushu University, Japan \\ ${ }^{\mathrm{b}}$ Faculty of Engineering, Kyushu University, Japan
}

\begin{abstract}
The use of underwater robots and vehicles for marine observation, undersea work, and the development of seabed resources has increased in recent years. One such robot is the fish-type robot, which swims with fins instead of thrusters. The fishtype robot developed by Kyushu University uses artificial muscles instead of motors. However, the power of the artificial muscle used in the fish-type robot is lower than that of a motor, meaning the driving force of the fish-type robot is lower than that of a robot using motors. To solve this problem, a fish-type robot that moves its body using servo motors and moves its fins using artificial muscles was developed. Several kinds of swimming performances of the fish-type robot were examined by experiments and calculations.

Additionally, the fish-type robot does not produce suction flows in contrast to typical underwater vehicles. It has been inferred that animals living in the water are not harmed in any way by the swimming mechanism. The proposed fish-type robot and a common thrustertype vehicle were driven in a water tank containing a large amount of imitation seaweed. The two devices were driven under the same conditions, and the effects of obstacles in water were studied.
\end{abstract}

Index Terms-Fish-type robot, Artificial muscle, Servo motor, Biomimetic robot, Swimming mechanism, DC motor, Thruster.

\section{INTRODUCTION}

In recent years, the use of underwater robots and vehicles for marine observation, undersea work, and the development of seabed resources has increased. One such robot is the fish-type robot, which swims with fins instead of thrusters.

The fish-type robot developed by Kyushu University uses artificial muscles instead of motors [1]. In this way, this robot can move more quickly than a robot using motors. In addition, because the structure of artificial muscles is simple, little space is required for the actuators.

However, the power of the artificial muscle used in the fish-type robot developed by Kyushu University is lower than that of a motor, meaning the driving force of the fish-type robot is lower than that of a robot using motors. In actual fish, muscles are distributed throughout the body. In contrast, most fish-type robots developed thus far are equipped with actuators clustered in a small region within the robot body. This is one of the reasons that swimming ability of those robots is inferior to that of actual fish.

Considering these points, to achieve high movement performance in water, a fish-type robot that moves its body using servo motors and moves its fins using artificial muscles was developed in this study. Its artificial muscles were used during the quick turn maneuver. In the past studies, only servo motors were used in the quick turn maneuver of fish-type robot [2, 3]. In the study of Koichi HITARA and others, the momentary angular velocity of the fish-type robot was about $100 \%$ in quick turn maneuver. In the study of Hideaki FURUKAWA and others, the calculated average angular velocity was $90 \%$ s and the measured that was $40 \% \mathrm{~s}$ in quick turn maneuver.

In this paper, both artificial muscles and servo motors were used in the quick turn maneuver at the same time. The performances of the developed fish-type robot such as advance and quick turn were then assessed by experiments and calculations.

In contrast to typical underwater vehicles, the fishtype robot does not produce suction flows using thrusters. It has been inferred that animals living in the water are not harmed in any way by the swimming mechanism. Additionally, foreign substances often become entangled in the shafts of underwater vehicle thrusters. Fish-type robots using a biomimetic swimming mechanism can avoid these problems associated with thrusters. Therefore, the proposed fishtype robot and a common thruster-type vehicle were driven in a water tank containing a large amount of imitation seaweed. The two devices were driven under the same conditions, and the number of trials in which imitation seaweed became entangled in the two devices was determined. 


\section{DEVELOPMENT OF FIN-TYPE ACTUATOR}

\section{A. Driving method}

This paper proposes a swimming mechanism using artificial muscles and servo motors as actuators to drive a fish-type robot. The rear of the body is driven by servo motors, and the tail fin, two pectoral fins, dorsal fin, and anal fin, which are made of polystyrene chloride, are driven by artificial muscles. The arrangement of the fish-type robot is shown in Fig. 1.

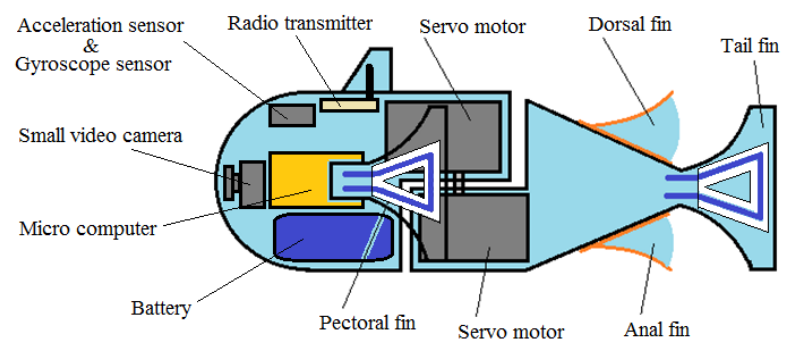

Fig. 1 Arrangement of fish-type robot

Two servo motors were installed in one joint at the central part of the body, and the tail fin, pectoral fins, dorsal fin, and anal fin, all of which include artificial muscles, were attached to the body. Because of the use of the two servo motors, the maximum angular velocity and the range of the drive angle on the joint of the present fish-type robot are twice that in the case using one motor.

The motion of the fish-type robot using only the servo motors was compared with that using the servo motors and artificial muscles. Actual fish use their pectoral fins to turn and stop $[4,5]$. During quick turning maneuvers, the body undergoes yaw rotation by producing drag on one side. The motion of the robot using only the servo motors during quick turning maneuvers was compared with that of using the servo motors and artificial muscles to confirm the maximum angular velocity.

The tail and pectoral fins are driven in the yaw direction, and the dorsal and anal fins are driven in the pitch direction. The drag coefficient in the relative flow direction when the fish-type robot turns quickly is increased by the use of dorsal and anal fins. This increases the rotational moment acting on the body, causing the inertial force acting on the fins to decrease and the drag acting on the fins to increase because the fins are flat and lightweight. Therefore, the turning angle of the robot increases. The structure and driving image of each fin during a quick turn maneuver are shown in Fig. 2. The $x$-, $y$-, and $z$-axes are in the direction of the length, width, and height of the robot body, respectively. Its fins were made of artificial muscle, polystyrene chloride sheet and silicon sheet. Those were joined with adhesive. Acryl-modification silicone resin was used as the adhesive.

The pectoral and tail fins produce drag. This drag is large relative to the inertial force produced by the pectoral and tail fins. The dorsal and anal fins increase the projected area normal to the flow direction and the drag coefficient for the back half of the robot. The performance of the robot when executing quick turning maneuvers increases when these fins are spread.

\section{B. Deformation of fins by artificial muscles}

The fins of actual fish deform into various shapes when the fish swim underwater. The structure of the fins and a conceptual diagram describing the deformation of the fin by the artificial muscle are shown in Fig. 3. The $d l$ is micro length in the fin.

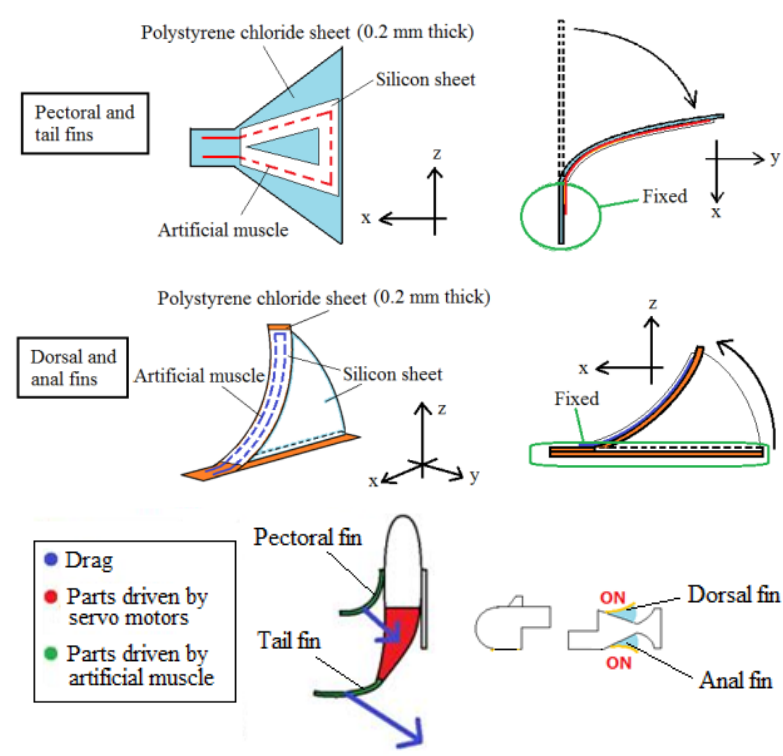

Fig. 2 Structure and driving image of the each fin during a quick turning maneuver (a)

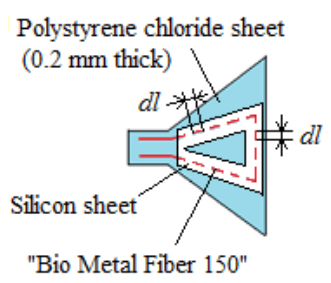

(b)

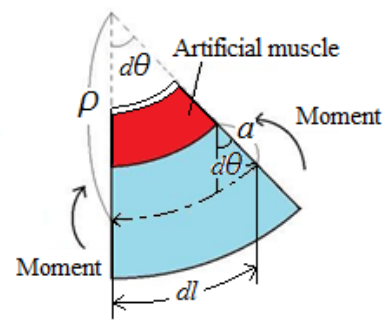

Fig. 3 (a) Attachment of artificial muscle to the fins and (b) conceptual diagram of deformation in the $d l$-cross section of the fins

The artificial muscle is attached to each fin, as shown in Fig. 3 (a). The conceptual diagram in Fig. 3 (b) shows the $d l$-cross section of the fin.

The fiber-like actuator Bio Metal Fiber 150 (Toki Corporation, Inc.) was used for the artificial muscles. Bio Metal Fiber 150 is $150 \mu \mathrm{m}$ in diameter, and a length of $11 \mathrm{~cm}$ was used for each fin. This artificial muscle can be deformed and restored to its original condition by switching the electricity on and off, respectively. The strain $\varepsilon$ of the artificial muscle and the radius $\rho$ of the curvature of the fins are given by

$$
\varepsilon=a \frac{\mathrm{d} \theta}{\mathrm{dl}}=\frac{a \mathrm{~d} \theta}{\rho} \frac{a}{\mathrm{~d} \theta}=\frac{a}{\rho}
$$




$$
\rho=\frac{a}{\varepsilon}=\frac{a L}{\Delta L}
$$

where $a$ is half of the thickness of the fin, $\mathrm{d} \theta$ is an infinitesimal angle, $d l$ is an infinitesimal length, $L$ is the length of the artificial muscles, and $\Delta L$ is the contraction in the length of the artificial muscles. The setup of the experiment for measuring the shrinkage of the artificial muscle is shown in Fig. 4.

The artificial muscle was covered with an adhesive to attach it to the surface of the fin. The voltage of electromotive force of the circuit in Fig. 4 is $11.1 \mathrm{~V}$.

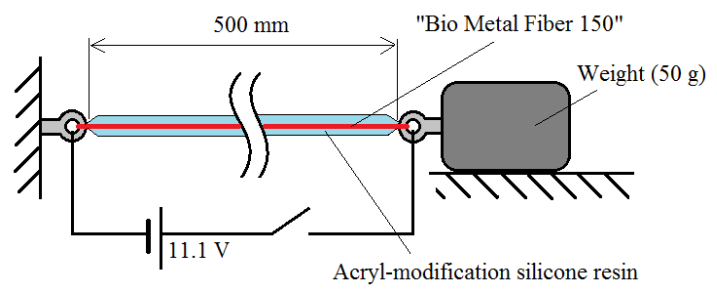

Fig. 4 Experiment for measuring the contraction of the artificial muscle

This voltage was applied to the circuit of the fish-type robot by a battery installed in the robot. In this experiment, $\Delta L$ was $0.24 \%$ of the original length $L$ of the artificial muscle.

The deformation of the fins was calculated using $\Delta L$. The deformation of the trailing edge of the fin is shown in Fig. 5.
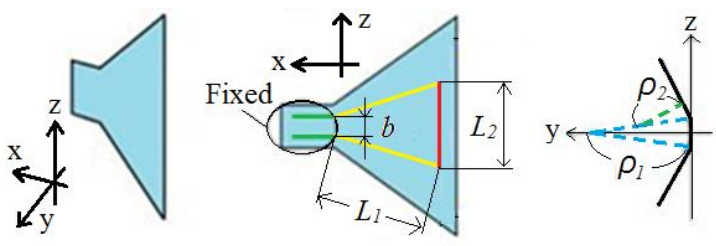

Fig. 5 Estimated trailing edge shapes of fins

The deformations of the trailing edge shapes are given by

$$
\begin{gathered}
\rho_{1}=\frac{a L_{2}}{\Delta L_{2}} \\
\rho_{2}=\frac{2 a L_{1 z}}{2 \Delta L_{1 z}+\Delta L_{2}-\Delta b}
\end{gathered}
$$

where $L_{1}$ and $L_{2}$ are the two variable dimensions of the artificial muscle, as shown in Fig. $5 ; b$ is the distance between the two ends of the artificial muscle, which is fixed; $L_{1 z}$ is the length of the projection of $L_{1}$ onto the $z$ axis; $\Delta L_{1 z}$ is the contraction of $L_{1}$ in the $z$-direction; $\Delta b$ is the contraction of $b$; and $\rho_{1}$ and $\rho_{2}$ are the radii of curvature deformed by the change in $L_{2}$ only and by the changes in both $L_{1}$ and $L_{2}$, respectively. The deformations of the fins in air and water are shown in Fig. 6. Here, the tail fin angle from the centerline is 45 degrees as shown in the figure. The electricity supplied to the artificial muscles on the fins, was switched on for $0.1 \mathrm{~s}$. And the motion of the fin was photographed with high speed mode of a camera. The calculated and measured trailing edge shapes are shown in Fig. 7. (a)

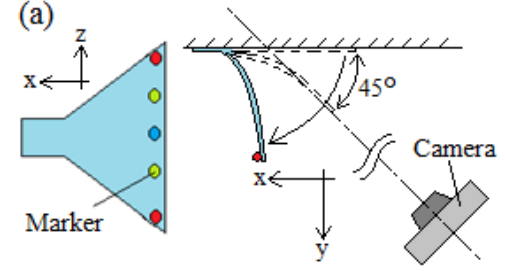

(b)

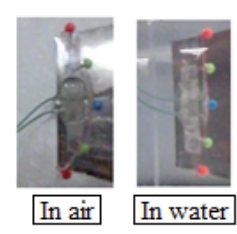

Fig. 6 (a) Coordinate system of the fin and (b) trailing edge shapes of fins deformed in air and water

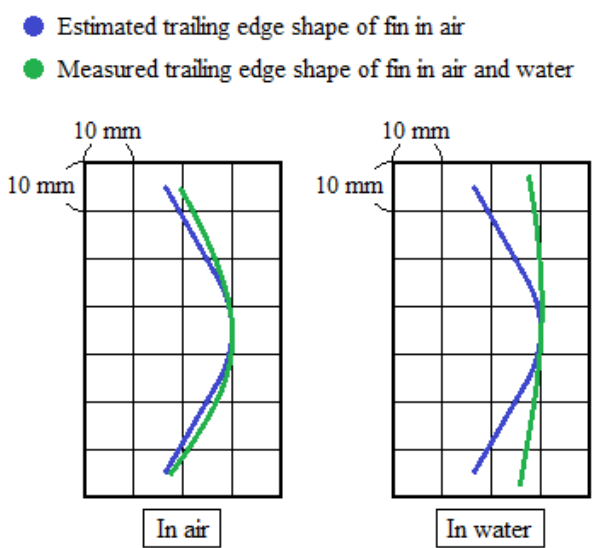

Fig. 7 Estimated and measured trailing edge shapes of fins deformed in air and water

The calculated and actual shapes of the fins deformed in the air were found to be in good agreement. The measured shape of the fin deformed in the water was affected by fluid force. Based on an examination of the drag coefficients of various shapes, it was predicted that the largest drag occurs when a fin is flat. The shape of a fin underwater was approximately flat in this experiment, as shown in Fig. 7; therefore, it is thought that this shape is suitable for producing a large drag.

\section{Fluid force acting on fins}

The original shape of the fins was then improved to increase the drag. The drag of the fin during electricity was supplied to the artificial muscles was measured by a three component force sensor. The original and improved fin shapes are shown in Fig. 8, and the drag forces acting on the fins are shown in Fig. 9. The drag acting on the improved fin was greater than that acting on the original fin because the area of the fin was concentrated around the tip of the fin where the relative velocity would be greatest. 


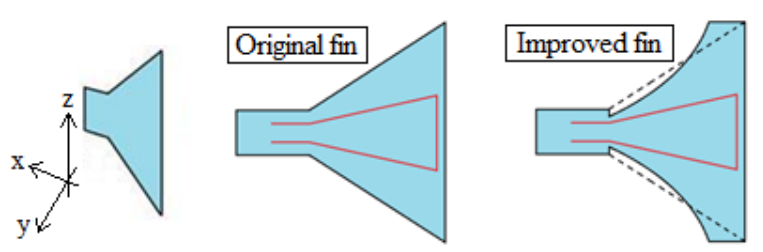

Fig. 8 Original and improved fin shapes
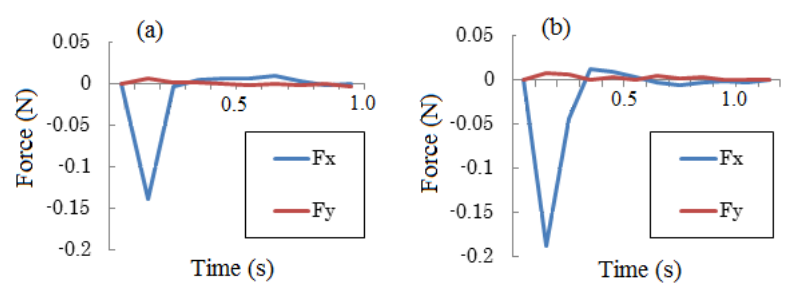

Fig. 9 Drag forces acting on the (a) original and (b) improved fins

The microcontroller mbed NXP LPC1768 (NXP Semiconductors) was used to control the motion of the fish-type robot. Xbee PRO (Digi International) was adopted as a radio transmitter to check the operation of the fish-type robot from on shore. A lithium polymer battery with an output of $11.1 \mathrm{~V}$ was used as the battery. A photograph of the fish-type robot is shown in Fig. 10. The length of the robot is $258 \mathrm{~mm}$, and its weight in air is $394 \mathrm{~g}$.

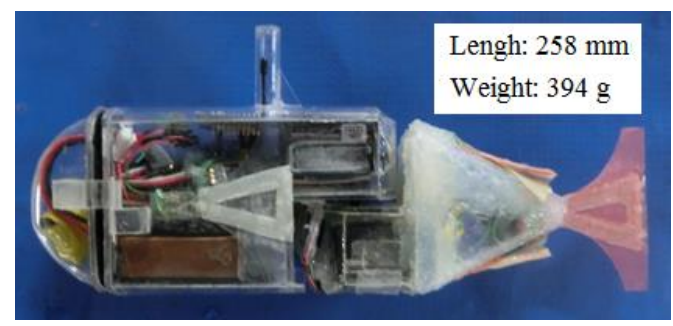

Fig. 10 Fish-type robot using artificial muscles and servo motor

\section{SWIMMING PERFORMANCE OF FISH-TYPE ROBOT}

The swimming performance of the developed fish-type robot with the improved fin shape was experimentally evaluated. Photographs of the fish-type robot swimming in a water tank were analyzed to evaluate its performance.

The maximum forward speeds of the robot were measured at time of drive cycle of $0.25,0.3,0.4,0.5$, and $0.6 \mathrm{~s}$. The control signal used to make the robot swim in a straight trajectory is shown in Fig. 11. The control signal in Fig. 11 is the sum of the yawing angle of two servo motors during straight swimming when two servo motors are called "Servo 1" and "Servo 2". This control signal is the signal that was used in a previous study [6].

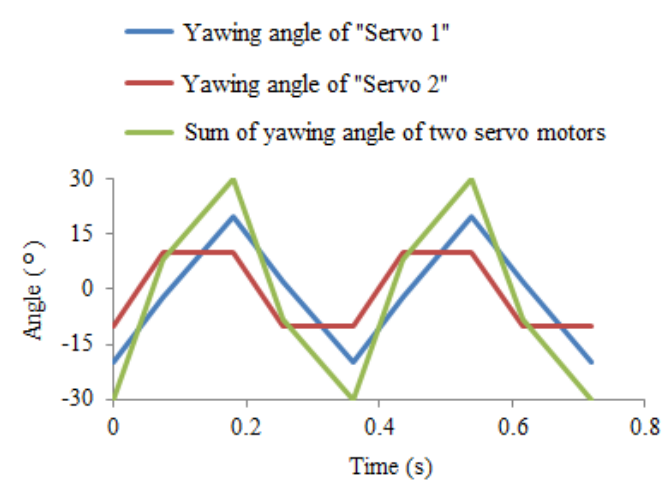

Fig. 11 Control signal of two servo motors during straight swimming

The equations to obtain the driving force of the fishtype robot are based on slender body theory [7-13] and are given by

$$
\begin{aligned}
T=-\frac{\partial}{\partial t} \int_{-l n}^{l t} & \rho_{w} \pi b_{(x)}{ }^{2} w \frac{\partial h}{\partial x} d x \\
& +\left[\rho_{w} \pi b_{(x)}{ }^{2}\left\{\frac{1}{2} w^{2}-U w \frac{\partial h}{\partial x}\right\}\right]_{x=l t} \\
& -\int_{0}^{l m}\left\{\frac{1}{2} w^{2}-U w \frac{\partial h}{\partial x}\right\} \rho_{w} \pi \frac{\partial b_{(x)}^{2}}{\partial x} d x \\
& +\int_{l m}^{l t}\left\{\frac{1}{2}\left(w-[w]_{x=x^{*}}\right)^{2}-\frac{1}{2} w^{2}\right. \\
& \left.+U[w]_{x=x^{*}} \frac{\partial h}{\partial x}\right\} \rho_{w} \pi \frac{\partial b_{(x)}}{\partial x} d x \\
h_{(x, t)} & =f_{(x)} g(t-x / c) \\
w_{(x, t)} & =\left(\frac{\partial}{\partial t}+U \frac{\partial}{\partial x}\right) h_{(x, t)} \\
x^{*}= & x-U\left(t-t^{*}\right)
\end{aligned}
$$

where $l_{n}, l_{m}$, and $l_{t}$ are the $x$-coordinates of the top of the head, the location of the minimum height of the body, and the end of the tail fin, respectively; $T$ is the thrust; $h_{(x, t)}$ is the $y$-component of the displacement of the body; $f_{(x)}$ is the distribution of the amplitude of the motion of the body at each $x$-coordinate; $g$ is a periodic function; $c$ is the wave phase speed; $\rho_{w}$ is the density of water; $b_{(x)}$ is the distribution of the height in the $x$ direction; $U$ is the speed of the flow around the fishtype robot; and $t^{*}$ is the time when a vortex is released at the tail fin. The origins of the $x-, y$-, and $z$-axes are the location of the maximum height of the body, the center of the body in the width direction, and the bottom of the body, respectively. The positive direction of $U$ is toward the rear of the robot.

Next, an experiment was conducted to demonstrate the quick turning performance of the fish-type robot. The robot executed the quick turning maneuver in $0.62 \mathrm{~s}$. 
The arms of the servo motors were moved $90^{\circ}$ in the direction of the turn, then back to their original positions. The control signals of the two servo motors and each fin during the quick turn experiment are shown in Figs. 12 and 13, respectively.

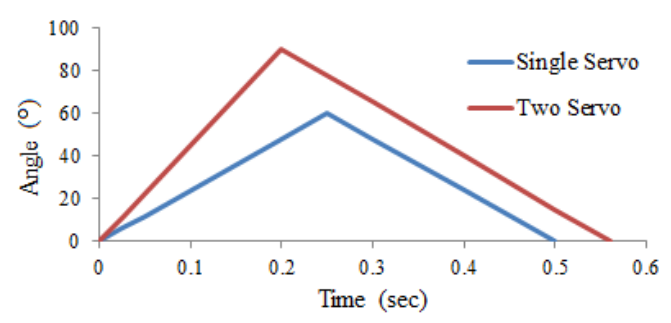

Fig. 12 Control signals of the two servo motors during quick turn experiment
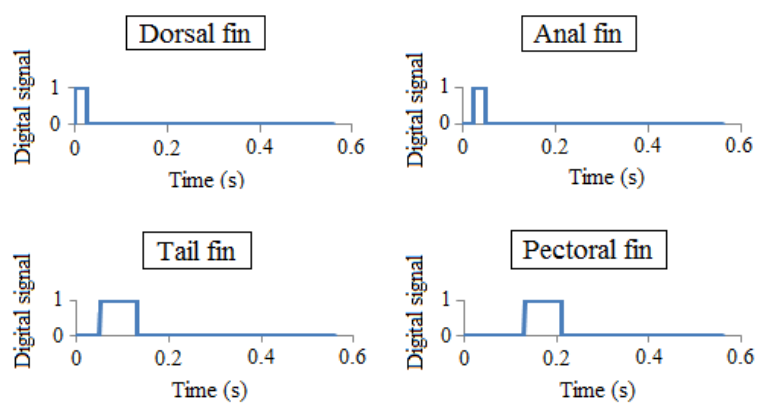

Fig. 13 Control signals of the fins during the quick turn experiment

The two servo motors were driven simultaneously when the arms of the servo motors were moved $90^{\circ}$ in the direction of the turn. They were then driven at different times when their arms returned to their original positions. The fins were also driven at different times during the quick turning maneuvers because the electric current sent to the artificial muscle is dispersed if two fins are driven simultaneously. The dorsal and anal fins were driven earlier than the tail and pectoral fins, because the dorsal and anal fins must be driven in the early stage of a turn to get enough effect.

In the quick turn experiment, two cases were investigated: motion driven by the fins and servo motors and motion driven by only the servo motors. In addition, the results of sequential quick turning maneuvers were evaluated.

The measured maximum forward speed of the robot and the calculated average thrust during swimming are shown in Fig. 14.

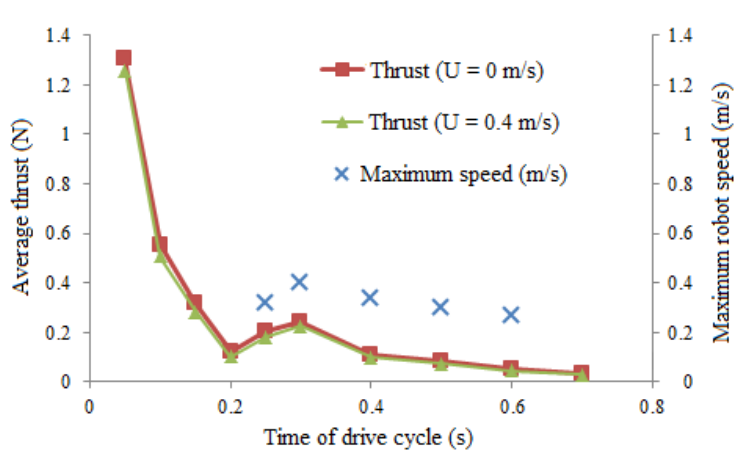

Fig. 14 Maximum forward speed and calculated average thrust plotted against time of drive cycle

As shown in Fig. 14, the maximum thrust was attained in the time of drive cycle of $0.3 \mathrm{~s}$. In this case, the measured maximum forward speed was approximately $0.4 \mathrm{~m} / \mathrm{s}$, and the robot reached the maximum speed $2 \mathrm{~s}$ after it began swimming.

The yaw rotation during a quick turn to the left was $38.7^{\circ}$ with only the two servo motors and $48.8^{\circ}$ with the two servo motors and the fins. Similarly, the yaw rotation during a quick turn to the right was $33.8^{\circ}$ with only the two servo motors and $45.2^{\circ}$ with the two servo motors and the fins. Therefore, the average angular velocity of the robot using only the two servo motors was $62.4 \%$ and $54.5 \%$ when turning to the left and right, respectively, whereas that using the two servo motors and the fins was $78.7 \%$ s and $72.9 \%$ s when turning to the left and right, respectively. The average angular velocity increases during the quick turning maneuver using the artificial muscles, because the drag around the fish-type robot during driving the fins was higher than that without fins. It is believed that the reasons of this improvement are the increase of the relative current speed around the fins and the projection area of the body.

When the robot using the two servo motors and the fins made three consecutive quick turns, the yaw rotation was $167^{\circ}$, and the average angular velocity was $115 \%$ s. Therefore, the robot can turn around in approximately $1.6 \mathrm{~s}$.

An acceleration sensor and an angular velocity sensor were attached to the robot. The acceleration sensor was used to measure the acceleration of the robot in the plane direction. The velocity of the robot was then calculated by integrating the acceleration data. When the robot inclines, the acceleration due to gravity was added to the acceleration data. Based on the attitude of the robot, which was measured using the angular velocity sensor, the acceleration due to gravity was deducted from the original acceleration data.

The fish-type robot was driven forward in the water tank with no flow to confirm the effectiveness of the acceleration sensor. The acceleration measured by the sensor is shown in Fig. 15, and the speed calculated by integrating the acceleration data is shown in Fig. 16. The sway and surge velocities of the robot were approximately 0 and $0.45 \mathrm{~m} / \mathrm{s}$, respectively. It was confirmed that the estimated speed was nearly equal to 
that calculated by integrating the acceleration data, demonstrating the accuracy of the acceleration sensor.

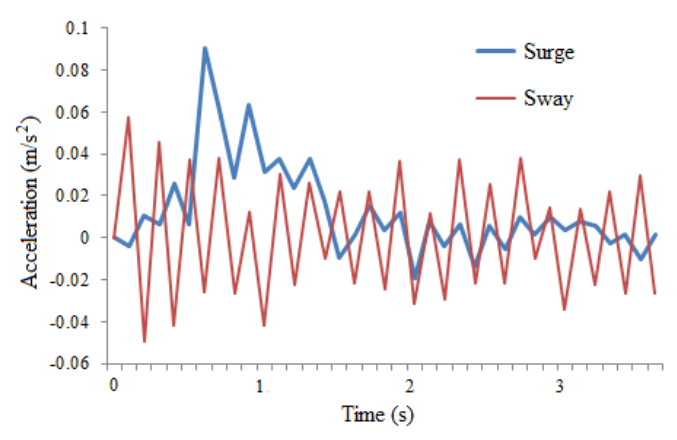

Fig. 15 Acceleration of the fish-type robot in a water tank with no flow

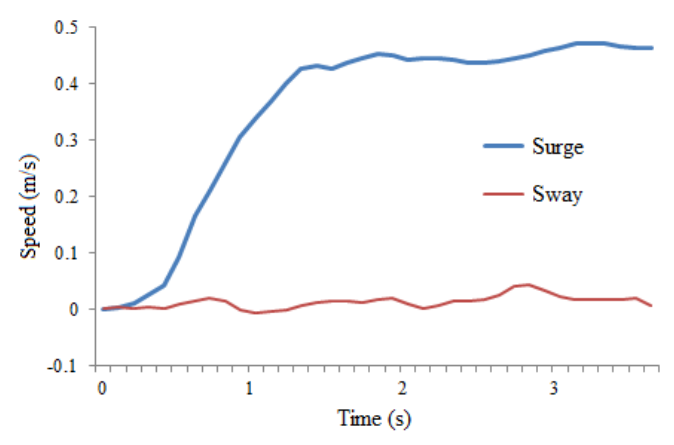

Fig. 16 Velocity of the fish-type robot in a water tank with no flow

Next, a control experiment was performed in the tank. The robot was pushed with a stick once to move it using an external force. The movement of the controlled robot under this disturbance was recorded by a video camera. The acceleration of the fish-type robot measured in this experiment is shown in Fig. 17, and the speed calculated by integrating the acceleration is shown in Fig. 18.

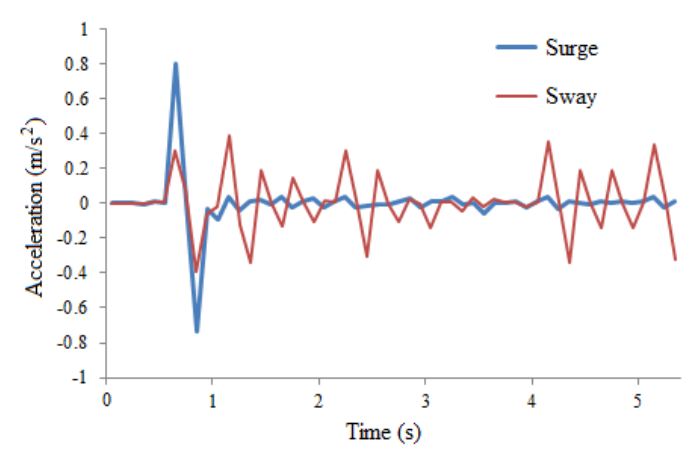

Fig. 17 Acceleration of the fish-type robot in the control experiment

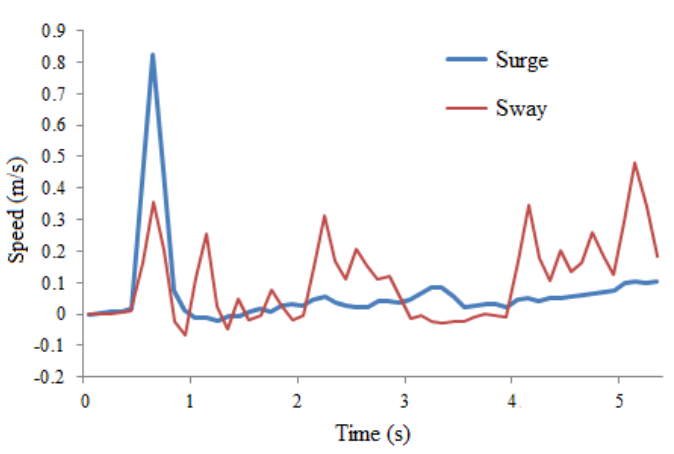

Fig. 18 Velocity of the fish-type robot in the control experiment

The surge and sway velocities did not converge after the disturbance because of the accumulation of acceleration sensor error. Another sensor must be used together with the acceleration sensor to realize sufficient control performance.

\section{EXPERIMENTS IN WATER WITH FOREIGN SUBSTANCES}

In contrast to typical underwater vehicles, the fishtype robot does not produce suction flows using thrusters. The suction flow caused by a rotating thruster is almost a steady flow. In contrast to that, the motion of the fish-type robot during advance is the reciprocating motion. Therefore, the fish-type robot does not produce large suction flows around the body. It has been inferred that animals living in the water are not harmed in any way by the swimming mechanism. Additionally, foreign substances often become entangled in the shafts of underwater vehicle thrusters. Fish-type robots using a biomimetic swimming mechanism can avoid these problems associated with thrusters.

The proposed fish-type robot and a common thrustertype vehicle were driven in a water tank containing a large amount of imitation seaweed. The two devices were driven under the same conditions, and the number of trials in which imitation seaweed became entangled in the two devices was determined. The imitation seaweed is made of polyethylene and floats on the surface of the water because its specific gravity is less than that of water. Pieces of imitation seaweed with a total mass of $4.8 \mathrm{~kg}$ were cast into the water tank. The surface of the water is $2 \mathrm{~m} \times 3 \mathrm{~m}$. Photographs of the imitation seaweed and the water tank containing the imitation seaweed are shown in Fig. 19. An arrangement and a photograph of the thruster-type vehicle are shown in Fig. 20. The length of the vehicle is $365 \mathrm{~mm}$, its weight in air is $792 \mathrm{~g}$, and its maximum forward speed is approximately $0.46 \mathrm{~m} / \mathrm{s}$. 

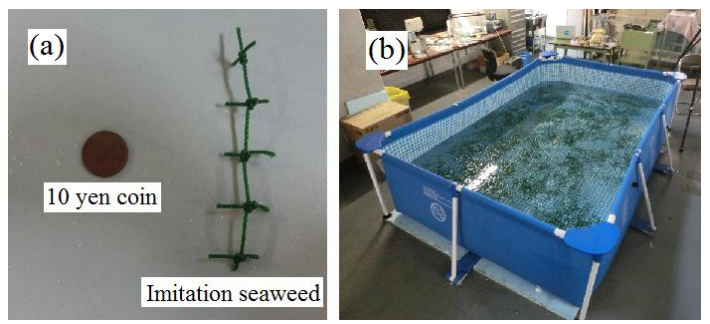

Fig. 19 (a) Imitation seaweed and (b) a water tank containing imitation seaweed

The two robots were driven lengthwise across the water tank 50 times (10 sets of five trials each). Then the number trials in which imitation seaweed became entangled in each device was counted. A conceptual diagram of this experiment is shown in Fig. 21.

(a)
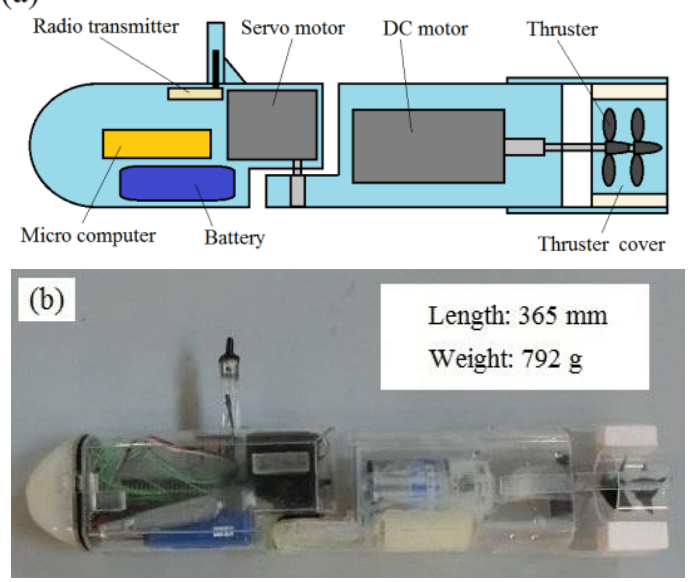

Fig. 20 (a) Arrangement and (b) photograph of thruster-type vehicle, which has a thruster attached to the body

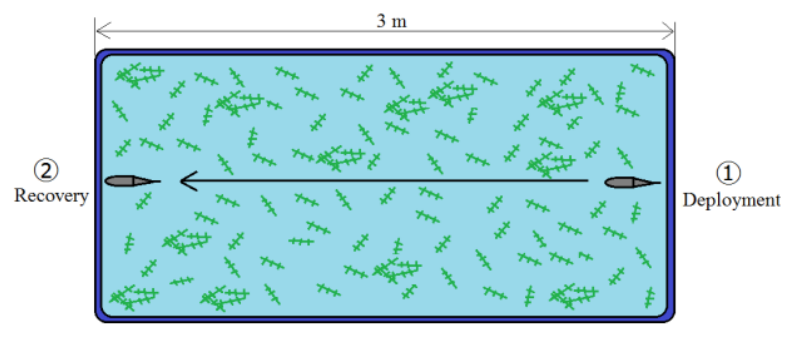

Fig. 21 Conceptual diagram of the experiment with imitation seaweed

In this experiment, imitation seaweed became entangled in each device in 27 of the 50 trials for the thruster-type vehicle and 0 of the 50 trials for the fishtype robot. Thus, the probability of seaweed becoming entangled in the thruster-type vehicle is $54 \%$ (27 of the 50 trials) and that of the fish-type robot is $0 \%$ ( 0 of the 50 trials). The robustness of the swimming mechanism of the fish-type robot in water containing foreign substances was thus confirmed.

It is predicted that the imitation seaweed is entangled with each fins and protrusions of the body. On the other hand, it is thought that animals living in the water are not harmed by the swimming mechanism of the fishtype robot at least. They can avoid from the swimming mechanism easily because the fish-type robot does not produce large suction flows.

\section{CONCLUSIONS}

This paper presented a novel swimming mechanism for a fish-type robot using artificial muscles and servo motors. Fins using artificial muscles were developed to improve the quick turning performance of the robot. Then, these fins and two servo motors were installed in the robot, and the swimming performance of the robot was evaluated.

Slender body theory was used to estimate the thrust of the robot. The maximum thrust was attained when the time of drive cycle was $0.3 \mathrm{~s}$. In this case, the measured maximum forward speed of the robot was approximately $0.4 \mathrm{~m} / \mathrm{s}$, and the robot reached the maximum speed in $2 \mathrm{~s}$ after the start.

During quick turning maneuvers, the motion of the robot driven by only the servo motors was compared with that driven by the servo motors and artificial muscles to confirm the maximum angular velocity in the motion. the average angular velocity of the robot using only the two servo motors was $62.4 \%$ and $54.5^{\circ} \%$ when turning to the left and right, respectively, whereas that using the two servo motors and the fins was $78.7 \% \mathrm{~s}$ and $72.9 \%$ when turning to the left and right, respectively. Additionally, the average angular velocity during quick turning maneuver was $115 \%$ s by consecutive movement. In the past studies about fishtype robot with only servo motors, the angular velocity does not reach $115 \%$ s $[2,3]$.

Next, an acceleration sensor and an angular velocity sensor were attached to the fish-type robot, and a control experiment was performed in a water tank. The surge and sway velocities did not converge after the robot experienced an external disturbance because of the accumulation of the acceleration sensor error.

Finally, the fish-type robot and a thruster-type underwater vehicle were driven in a water tank containing imitation seaweed, and the number of trials in which imitation seaweed became entangled in each of the devices was determined. The imitation seaweed became entangled in each device in 27 of the 50 trials for the thruster-type vehicle and in 0 of the 50 trials for the fish-type robot. Thus, the probability of seaweed becoming entangled in the thruster-type vehicle is $54 \%$ (27 of the 50 trials) and that of the fish-type robot is $0 \%$ ( 0 of the 50 trials). The robustness of the swimming mechanism of the fish-type robot in water containing foreign substances was thus confirmed. 


\section{REFERENCES}

[1] S. Yamaguchi, K. Kagiyama, and M. Terada, "Development of a motion mechanism for fish type robots", The Japan society of Naval Architects and Ocean Engineers, vol. 1, pp. 27-34, 2006.

[2] K. Hirata, K. Harumi, T. Takimoto, K. Tamura, M. Makino, Y. Kodama and H. Tomita, "Fundamental Study on Fish Robots", National Maritime Research Institute, vol. 2, no. 3, pp. 281-307, 2002.

[3] H. Furukawa, A. Konno, K. Hirata, Y. Niki and M. Kawada, "A study on quick-turning of submersible robot with caudal fin", The Japan Society of Mechanical Engineers, vol. 6, no. 9, pp. 153-154, 2009.

[4] N. Kato and H. Suzuki, "Motion of Pectoral Fin of Fish and Its Application to Underwater Robots", The Japan Society of Fluid Mechanics, vol. 12, pp. 143-153, 2005.

[5] T. Ariyoshi, N. Kato, H. Suzuki, Y. Ando, K. Suzumori, T. Kanda, and S. Endo, "Elastic Pectoral Fin Actuator for Biomimetic Underwater Vehicle", The Japan Society of Naval Architects and Ocean Engineers, vol. 5, pp. 15-25, 2007.

[6] S. Fujiwara and S. Yamaguchi, "Development of a Fish Type Robot using Artificial Muscles and Servo Motors", Society of Aero Aqua Bio-mechanisms, vol. 33, pp. 2526, 2015.

[7] R. T. Jones, "Properties of Low Aspect Ratio Pointed Wings at Speeds Below and Above the Speed of Sound", NACA, Rep. 835, 1946.

[8] J. R. Spreiter, "Aerodynamic Properties of Slender Wing-Body Combinations at Subsonic, Transonic, and Supersonic Speeds", NACA, TN 1662, 1948.

[9] J. Weber, D. A. Kirby and D. J. Kettle, "An Extension of Multhopp's Method of Calculating the Spanwise Loading of WingFuselage Combinations", British A.R.C, R\&M 2872, 1951.

[10] M. J. Lighthill, "Note on the Swimming of Slender Fish", J. Fluid Mech, vol. 9, pp. 305317, 1960.

[11] M. J. Lighthill, "Hydrodynamic of Aquatic Animal Propulsion", Anm.Rev.Fluid Mech, vol. 1, pp. 413-446, 1969.

[12] M. J. Lighthill, "Aquatic Animal Propulsion of High Hydromechanical Efficiency”, J. Fluid Mech, vol. 44(2), pp. 265-301, 1970.

[13] M. J. Lighthill, "Large-Amplitude ElongatedBody Theory of Fish Locomotion", Proc.Roy.Soc.London, vol. 179, pp. 125-138. 1971. 